\title{
Análisis del desempeño de la gestión administrativa y operacional del Laboratorio Clínico del Hospital de la Anexión
}

\author{
Ana María Mora Vicarioli ${ }^{1}$ \& Rafael Rivero Soto² \\ 1. Enfermera. Corporación Oftalmológica, Sabana Sur, San José. Correo electrónico: vicarioli8@hotmail.com, \\ Cel.: 8344-0139. \\ 2. Microbiólogo. Laboratorio Clínico Hospital de la Anexión, Guanacaste. Caja Costarricense del Seguro Social. \\ Correo electrónico: rafarivero1345@gmail.com, Cel.: 8832-2864.
}

Recibido: 09 mayo 2016

\section{RESUMEN}

La realización de este proyecto permitió obtener una valiosa información de los procesos en las diversas áreas del Laboratorio Clínico del Hospital de la Anexión, permitiendo medir el nivel de desempeño para conformar una herramienta en el proceso de gestión. Se evidenció una baja en el desempeño en la cantidad de personas usuarias atendidas y por consiguiente de análisis realizados en el mes de mayo de 2011 con respecto al promedio esperado. Se evidenció la existencia de debilidades en el funcionamiento del laboratorio y particularmente en la atención directa al paciente. El usuario del laboratorio se encuentra satisfecho en lo que respecta al trato que recibe. Toda esta información sirvió de base para la confección de las debidas recomendaciones con el fin de que se puedan tomar acciones para elevar el nivel de desempeño y para la toma de decisiones a futuro.

Palabras clave: Control de gestión, eficiencia, eficacia, calidad, laboratorio clínico.

\section{ABSTRACT \\ Performance analysis of administrative and operational management of the Laboratorio Clínico del Hospital de la Anexión}

The completion of this research yielded valuable information regarding the processes in different areas of the Clinical Laboratory of the Hospital de la Anexión, resulting in a management tool to measure the performance in every process. It showed a decline in performance on the number of people served and therefore of analysis conducted in the month of May 2011 compared to the expected average. These results suggest the existence of weaknesses in the lab's operations, particularly in direct patient care services. The lab user is satisfied with respect to the treatment received. All this information was the basis for making appropriate recommendations in order that action can
Aceptado: 16 mayo 2016

be taken to raise the level of performance and for future decision making.

Key words: management control, efficiency, effectiveness, quality, clinical laboratory.

\section{Introducción}

El presente estudio se realizó en el Laboratorio Clínico del Hospital de la Anexión (LCHA), ubicado en la Provincia de Guanacaste. La realización de este proyecto tuvo por objetivo general analizar el desempeño de la gestión administrativa y operacional del Laboratorio Clínico del Hospital de la Anexión (LCHA), Guanacaste, con respecto a la eficiencia, eficacia y calidad en el período comprendido entre Marzo y Julio de 2011.

Los objetivos específicos fueron:

- Determinar la eficacia y eficiencia y calidad de la gestión administrativa y operacional de del Laboratorio Clínico del Hospital de la Anexión, por medio de indicadores de capacidad resolutiva y de producción de análisis clínicos provenientes de consulta externa, así como los índices de satisfacción del usuario.

- Evaluar la eficiencia del desempeño de los colaboradores del laboratorio mediante indicadores de gestión de procesos sustantivos. 
- Formular las recomendaciones para el manejo integral y la mejora de la gestión en el LCHA.

Se consideró que la investigación fue accesible, así como la obtención de datos importantes de utilidad acerca de los servicios brindados. Se aplicaron indicadores de gestión como fuente de medición de la eficacia, eficiencia y calidad para analizar el desempeño de la gestión administrativa y operacional del laboratorio.

\section{Materiales y métodos}

Para efectos de esta investigación se utilizó el paradigma positivista. Con el fin de cumplir con los objetivos propuestos en la investigación, se empleó el enfoque cuantitativo. Este enfoque permitió describir cada variable por medio de datos estadísticos, para así obtener resultados que pudieran ser generalizados. Para la determinación se la población se utilizó el muestreo aleatorio sistemático (Robledo, 2004)

$$
\mathrm{n}_{0}=\frac{\mathrm{Z}_{\alpha} \mathrm{S}^{2}}{\mathrm{~d}^{2}}
$$

$\mathrm{n}_{0}$ : es el tamaño de muestra para una población infinita.

$\mathrm{Z}_{\alpha}$ : es un valor asociado al nivel de confianza, en este caso 1,64 para un nivel de confianza del $95 \%$.

S: es la desviación estándar, que en este caso sería la desviación máxima 50\%.

d: es el error máximo permitido, en este caso del $5 \%$.

Sin embargo, para fortalecer la investigación y poder analizar el desempeño de la gestión administrativa y operacional del laboratorio clínico se hizo necesario aplicar técnicas cualitativas como el cuestionario, la entrevista semiestructurada y lista de cotejo dirigida a informantes claves así como la observación no participante.

Este estudio fue de tipo transversal descriptivo ya que se recolectaron los datos en un momento dado y en un tiempo determinado y su objetivo central fue la descripción de fenómenos, ya que se seleccionaron una serie de variables que se midieron, con el fin de lograr analizar el desempeño de la gestión administrativa y operacional del LCHA.

Se utilizaron fuentes de información primarias entrevistando a los profesionales de cada sección así como fuentes secundarias (revistas, tesis y libros de texto, registros estadísticos y datos anuales de producción). También se tuvo acceso a los registros del personal en el Departamento de Recursos Humanos. Como herramienta para el análisis de los datos recopilados se utilizó un Cuadro de Operacionalización de Variables.

Este estudio estuvo orientado a la explicación, pues buscó respuestas a problemas concretos para la toma de decisiones a fin de tratar de mejorar las situaciones existentes, analizando el desempeño de la gestión administrativa y operacional del LCHA.

\section{Resultados}

La mayoría de las personas entrevistadas se encontraron en la adultez media constituyéndose en la etapa de mayor productividad dentro del ciclo vital, en contraste con un porcentaje menor de personas adultas mayores y menores de edad.

\section{Eficacia}

A pesar de que la mayoría de las personas encuestadas consideran oportuno el servicio del Laboratorio $(78,5 \%)$, si se pudo constatar que entre las principales deficiencias en el servicio se encuentra la atención directa a los usuarios, el horario y la fragmentación de la población para su atención.

\section{Eficiencia}

En lo que respeta a la eficiencia del desempeño de la gestión administrativa y operacional, las opiniones desfavorables giraron en torno al tiempo de espera para ser atendidos, desorden, impuntualidad e insuficiente personal para su atención.

Internamente los colaboradores manifestaron la necesidad de más personal para el óptimo desempeño por parte de cada uno de los 
empleados del laboratorio, redistribución de funciones y mejoras en la comunicación y el trabajo en equipo.

\section{Calidad}

En lo que respecta al trato al usuario, específicamente a la relación interpersonal establecida entre el personal del Laboratorio Clínico y los usuarios y usuarias, una mayoría (un 75,2\%) de los entrevistados refirieron estar de acuerdo con el trato recibido, sin embargo, los colaboradores concuerdan en que el trato a la persona usuaria constituye un aspecto en el cual existe espacio para crecer y mejorar.

Se pudo constatar que un porcentaje importante de la población considera excesivo el tiempo de espera para ser atendido, además manifiestan desinformación en cuanto al proceso.

En lo que respecta a las condiciones de infraestructura para la atención directa de los pacientes, un 56,8\% de los entrevistados manifestó estar insatisfecho ya que el espacio les resulta pequeño, obsoleto e incómodo. En cuanto al manejo del personal y de controles internos, se notó que se realizan esfuerzos importantes, sin embargo, se evidenció la falta de explotación de estos datos para una mejora en la gestión administrativa.

En cuanto a la concepción en general del servicio brindado, es considerado tanto por el personal como por el usuario, como bueno pero con posibilidades de mejorar siempre y cuando se les brinden las herramientas de recurso humano e infraestructura necesarias.

\section{Discusión}

Los laboratorios clínicos desempeñan un papel esencial en el diagnóstico, seguimiento de las alteraciones de la salud y vigilancia. En una institución prestadora de servicios de salud, tal como lo es el LCHA, es necesario mencionar la importancia del control de gestión, el cual es una de las herramientas fundamentales para que la institución pueda ofrecer adecuados servicios. El proceso de mejoramiento de los servicios de salud de Costa Rica, es un proceso continuo e interminable que debe ser constantemente monitoreado, en vista de que conjuga estadios de desarrollo cultural, tecnológico, científico y en lo fundamental humano, tanto para el que recibe los servicios como para el que los ofrece.

De acuerdo con Castillo (2009) la gestión, es por definición, la administración de procesos y toma de decisiones y la administración de costos y responsabilidades con un propósito definido. En consecuencia, es un proceso que conduce al cumplimiento de resultados los que a su vez son una característica implícita de la gestión. La expresión materializable de la gestión está en el diseño, la implementación, el control y la retroalimentación de los procesos.

El desempeño implica la consideración de un proceso organizacional, dinámico en el tiempo y refleja modificaciones del entorno organizativo (Hernández, 2002). Conforme se determinaron las conclusiones prioritarias, se perfilaron posibles recomendaciones por tomar en cuenta en esta unidad sanitaria.

Un aspecto fundamental por destacar es que, entre los sentimientos generados en los profesionales al momento de realizar la investigación, se encuentra la motivación, señal del interés por brindar una atención de calidad, eficiente y eficaz a esta población. Durante la aplicación de las entrevistas a los usuarios se pudo establecer un ambiente de confianza debido al compromiso, participación y sensibilización por parte de las profesionales, lo cual favoreció la construcción del conocimiento.

\section{Conclusiones y recomendaciones}

\section{Eficacia}

Se evidenció una disminución en la cantidad de personas usuarias atendidas y por consiguiente de análisis realizados, afectando la cobertura y la demanda actual del servicio. Los usuarios entrevistados se mostraron satisfechos con la atención y la normativa de atención de 250 pacientes diarios, situación que beneficia la organización del LCHA en cuanto a la cobertura. 


\section{Eficiencia}

La mayoría de las personas usuarias se encuentran en desacuerdo, en diferente medida, con el tiempo de espera para ser atendidos esto debido a la subutilización del personal asignado para atención directa y la limitación de las condiciones de infraestructura.

Existe incapacidad para hacerle frente al comportamiento variable e impredecible de la cantidad de personas que asisten diariamente a recibir el servicio del laboratorio clínico. Los pacientes entrevistados, así como el mismo personal encargado se encuentran en su mayoría estar en desacuerdo en cuanto a la cantidad de personal asignado para el proceso de la toma de muestra.

Se evidenció un problema en cuanto a la segmentación de la atención según su lugar de procedencia.

\section{Calidad}

El usuario de LCHA se encuentra satisfecho en cuanto al trato que recibe, sin embargo deben mejorarse las vías de comunicación y orientación durante el proceso de atención.

Existen limitaciones evidentes en cuanto a la infraestructura del área física dispuesta para la recepción, espera y atención directa de usuarios. Situación que condiciona negativamente la percepción del usuario acerca del servicio recibido.

Existe una mala distribución del espacio ya por si reducido e insuficiente del área de trabajo del laboratorio clínico en relación con la cantidad de personas que laboran en el primer turno específicamente.

No existe un encargado de la supervisión y análisis de los controles instaurados en los diferentes procesos que se llevan a cabo en el laboratorio lo que provoca un incorrecto aprovechamiento de los controles implementados, que permita el mejoramiento continuo y control de calidad.

La gestión de recurso humano resulta limitado en cuanto a la resolución de conflictos y estudio de puestos lo que compromete la calidad del trabajo dentro del LCHA.

\section{Recomendaciones}

\section{Eficacia}

Colocar un micrófono con parlantes en el área de la sala de espera con el objetivo de que las personas usuarias escuchen el número de la ficha al ser llamados para aligerar el proceso de toma de muestra.

Aumentar la cantidad máxima de personas atendidas por día a 300 usuarios, con una holgura superior del $20 \%$, sin llegar a comprometer la capacidad resolutiva ni de respuesta del laboratorio hacia el servicio de urgencias y hospitalización para brindar un mejor servicio.

\section{Eficiencia}

Promover la creación de nuevas plazas, con fundamento estadístico y demográfico, para garantizar la adaptación de la organización en un escenario dinámico en la zona y para cumplir con toda la demanda de las personas usuarias.

Reforzar la supervisión en todas las áreas del laboratorio variando la cantidad de personal de atención directa según la cantidad de usuarios para disminuir los tiempos de espera, realizando inspecciones matutinas en la zona de atención al paciente.

Diseñar un modelo de funcionamiento más flexible en cuanto a las funciones de los empleados, para afrontar diferentes escenarios según el volumen de trabajo, a cargo de la Jefatura del LCHA.

Planificar reuniones con el personal de manera periódica preestablecida con el fin de revisar el desempeño de los colaboradores ante las situaciones presentadas durante un tiempo determinado para el mejoramiento continuo del servicio.

Diseñar un plan de entrega de citas, a cargo de la Jefatura del LCHA, de acuerdo con las necesidades de los pacientes, no importa su 
procedencia proporcionando una mayor libertad para los usuarios.

\section{Calidad}

Colocar un buzón para la recepción de quejas y sugerencias, en la sala de espera de los pacientes para idear las acciones a seguir ante esas quejas y poder poner en práctica las sugerencias de los pacientes, con el fin de tener una ruta alterna de comunicación por escrito de los pacientes y un medio de retroalimentación para el mejoramiento continuo del servicio.

Se sugiere la supervisión continua en todas las áreas del laboratorio con el fin de evaluar la dinámica de trabajo del personal y la atención que se le brinda a los pacientes.

Mejorar los canales de comunicación del personal hacia los pacientes, no solo por medio de rótulos colocados en las paredes del Laboratorio, sino fomentando las buenas relaciones interpersonales entre personal y pacientes, esta labor debe estar a cargo de todos los colaboradores del Laboratorio.

Promover la creación de un sistema integrado de cómputo para el manejo de los resultados de los exámenes de Laboratorio, a cargo del Departamento de Informática de la Institución para disminuir los tiempos de entrega de resultados y que al mismo tiempo queden en base de datos para las estadísticas del LCHA.

Solicitar un estudio al departamento de mantenimiento del Hospital La Anexión, acerca de la posibilidad de hacer adaptaciones al espacio físico que se posee con el fin de mejorar la atención al público mejorando condiciones de iluminación dentro del área del laboratorio, la ventilación en el área de cubículos y área de espera.

Diseñar un protocolo de control de las bitácoras y documentación para así mejorar el control de procesos con el objetivo de alcanzar un mejoramiento continuo y la confección manual de calidad en que se especifique cada proceso, así como un control estadístico de loas mismos.

Se recomienda realizar continuamente actividades con el personal para mejorar la atención y el trato hacia la persona usuaria con capacitaciones cortas sobre diversos temas que ayuden a mejorar la calidad de atención, tales como: comunicación asertiva, manejo del estrés, trabajo en equipo, derechos y deberes, relaciones interpersonales.

\section{Referencias}

Alfaro, H. (2006). Análisis del proceso de recepción, análisis y entrega de los exámenes de hematología y química clínica del servicio de emergencias en el laboratorio clínico del Hospital Dr. Rafael Calderón Guardia durante los meses de agosto y setiembre del año 2006. (Tesis de Maestría). Universidad Estatal a Distancia, San José, Costa Rica.

Badilla, N. (2005). Análisis de los procesos sustantivos en el banco de sangre, laboratorio clínico, Doctor Clodomiro Picado, del Hospital San Juan de Dios y su relación con la eficiencia y eficacia en la prestación del servicio, en los meses de setiembre a diciembre, año 2004, San José. (Tesis de Maestría). Universidad Estatal a Distancia, San José, Costa Rica.

Barrantes, R. (1999). Investigación. Un camino al conocimiento. Un enfoque cualitativo y cuantitativo. San José: EUNED.

Bonnefoy, J. y Armijo, M. (2005). Indicadores de desempeño en el sector público Instituto Latinoamericano y del Caribe de Planificación Económica y Social - ILPES. Cepal, Santiago de Chile. Recuperado de http://www. eclac.org/publicaciones/xml/2/23572/manual45.pdf

Briozzo, G. y Perego, M. (2002). El gerenciamiento del laboratorio de análisis clínicos con la visión de calidad total. Revista del Hospital Materno Infantil Ramón Sardá, 2(1), 28-33.

Briozzo, G. y Perego, M. (2008). Fortalecimiento de la calidad: uso apropiado de la tecnología del laboratorio de análisis clínicos. Revista del Hospital Materno Infantil Ramón Sardá, 27(3), 124-127.

Caja Costarricense de Seguro Social (2008). Plan anual operativo 2009-2010 (PAO). Hospital la Anexión, Guanacaste. Laboratorio Clínico, CCSS.

Camacho, J. (2008). Nota estadística. Tamaño de muestra en estudios clínicos. Acta Médica Costarricense Colegio de Médicos y Cirujanos, 50, 20-21. 
Castillo, C. Vargas, B. (2009) El proceso de gestión y el desempeño organizacional. Cuadernos de Difusión. Volumen 4, Numero 26. 57-80.

Cope, D. (2000). Análisis de gestión administrativa de la Unidad de Terapia Respiratoria del Hospital Calderón Guardia, CCSS. (Tesis Maestría). Instituto Centroamericano de Administración Pública. Área de Proyectos de Desarrollo ICAP. San José, CR.

Deming, E. (1989). Calidad, productividad y competitividad: la salida de la crisis. Madrid: Ediciones Díaz de Santos.

Fuentes, M. y Rodríguez, R. (1994). El control de gestión como instrumento de optimización de la función pública. Anales de estudios económicos y empresariales, 9, 121-144.

García, R. (2004). Curso de Gestión Local de Salud para Técnicos del Primer Nivel de Atención. Caja Costarricense de Seguro Social. Recuperado de http:// www.cendeisss.sa.cr/cursos/sistemanacsaludgeneral. pdf

García, A. (2009). El análisis envolvente de datos, herramienta para la medición de la eficiencia en instituciones sanitarias, potencialidades y limitaciones. Revista Cubana de Higiene y Epidemiología. 47 (2), 1-10.

García, S. (1991). Nuevas actitudes y comportamientos empresariales. Madrid: Ediciones Díaz de Santos.

Gómez, Miguel. (2004). Elementos de Estadística Descriptiva. San José: EUNED.

González, P. (2004). Análisis del cumplimiento de los requisitos de acreditación en el Laboratorio Clínico del Hospital Nacional Psiquiátrico, en el primer cuatrimestre 2004. (Tesis de Maestría). Universidad Estatal a Distancia, San José, Costa Rica.

Gutiérrez, M. (2001). Administrar para la calidad. Conceptos administrativos del control total de calidad. México, D.F: Editorial Limusa.

Hernández, M. (2002). Evaluación del desempeño de las organizaciones públicas a través de la calidad. Ponencia presentada en el VII Congreso Internacional del CLAD sobre la Reforma del Estado y de la Administración Pública, Lisboa.
Hernández, R., Fernández, C. y Baptista, P. (1998). Metodología de la Investigación. (Segunda edición). México. D.F: McGraw Hill Interamericana.

Instituto Nacional de Estadística y Censos (INEC). (2010). Recuperado de http://www.inec.go.cr/

Instituto de Normas Técnicas de Costa Rica (INTECO). (2001). Compendio de Normas INTE-ISO 9000:2000, Sistemas de gestión de la calidad. Conceptos y vocabulario. San José: INTECO

Instituto de Normas Técnicas de Costa Rica (INTECO). (2005). Sistemas de gestión de la calidad-fundamentos y vocabulario. San José: INTECO.

Jiménez, G. y Valverde, L. (2008). Análisis del cumplimiento de los requisitos para la habilitación del Laboratorio Clínico del Área de Salud de Santo domingo: en búsqueda de la excelencia en calidad para el año 2007. (Tesis de Maestría). Universidad Estatal a Distancia, San José, Costa Rica.

Kaplan, R. y Norton, D. (2001). ¿Cómo utilizar el Cuadro de Mando Integral: para implantar y gestionar su estrategia? Barcelona: Gestión 2000.

Koontz, H., Weihrich, H. y Cannice, M. (2008). Administración. Una perspectiva global. México D.F: Mc Graw Hill.

Lemaitre, X. (1998). Normas ISO 9000: Alternativa de evaluación de la calidad en servicios de salud de hospitales de la CCSS. Análisis de caso: Laboratorio Clínico del Hospital México. (Tesis de Maestría). Instituto Centroamericano de Administración Pública, San José, Costa Rica.

Li, J. (1997). Diseño conceptual para el sistema de información automatizado como apoyo a la toma de decisiones en la consulta externa del Hospital de la Anexión, C.C.S.S. (Tesis de Maestría en Administración de Servicios de Salud Sostenibles). Instituto Centroamericano de Administración Pública, San José, Costa Rica.

Moya, M. (2000). Introducción a la estadística de la salud. San José: Editorial Universidad de Costa Rica.

Martin, G. y Murillo, C. (2004). Demanda intradiaria de un servicio de urgencias hospitalario. Análisis del comportamiento estacional. Cuadernos Económicos de la Información Comercial Española, 67, 107-133. 
Martín, N., Hernangómez J. y Martín, V. (2007). El deleite de la eficiencia. Universia Business Review, 14(5), 56-67.

Mokate, K. (2000). Diseño y gerencia de políticas y programas sociales. Banco Interamericano de Desarrollo, Instituto Interamericano para el Desarrollo Social. INDES. 2-3.

Organización Mundial de la Salud (OMS). (1976). Aplicación del análisis de sistemas a la gestión sanitaria. Informe de un comité de expertos de la Organización Mundial de la Salud. Serie de informes técnicos, N. 596.

Organización Mundial de la Salud (OMS). (2003). Informe sobre la salud en el mundo 2003, Forjemos el futuro. Recuperado de http://www.who.int/whr/2003/chapter7/es/index.html

Palmer, T. (1999). Definitions of efficiency. BMJ.

Pérez, M. (2003). El Sistema de Control de Gestión. Conceptos básicos para su diseño. Recuperado de http://www.gestiopolis.com/canales/gerencial/articulos/67/siscontrges.htm

Puig, J. (2000). Eficiencia en la atención primaria de salud: una revisión crítica de las medidas de frontera. Revista Española de Salud Pública. 74(5-6), 483-495

Robert, A. y Vijay, G. (2008). Sistemas de control de gestión. México: Mc Graw Hill/Interamericana Editores.

Robbins, S. y Coulter, M. (2005). Administración. México: Pearson Educación.

Robledo, J. (2004). Diseños de muestreos probabilísticos 11. Revista Nure Investigación, número, 11, 13-16.
Rodríguez, H. (2010). La gestión administrativa, respecto a las incapacidades laborales, otorgadas a funcionarios del Departamento de Mantenimiento, Lavandería y Servicios Domésticos del Hospital William Allen Taylor de Turrialba. San José, C.R.

Sáenz, L. (1998). Administración de Servicios de Salud. San José: EUNED.

Salvendy, G. (1982). Handbook of Industrial Engineering. United States: John Wiley and sons, Inc.

Sánchez, C. y Herrera, M. (2004). Satisfacción del usuario interno del servicio del laboratorio clínico de emergencias en los tres turnos, durante el tercer cuatrimestre del año 2004, en el Hospital San Juan de Dios, San José. (Tesis de Maestría). Universidad Estatal a Distancia, San José, Costa Rica.

Sanín, A. (1999). Control de gestión y evaluación de resultados en la gerencia pública. (Meta Evaluación- meso evaluación). Instituto latinoamericano y del Caribe de planificación económica y social-ILPES- Santiago de Chile.

Sarmiento, A. (2006). Análisis de eficiencia técnica de la red pública de prestadores de servicios dentro del sistema general de seguridad social en Salud. Revista Gerencia y Políticas de Salud, 5(11), 70-95.

Terry, G. y Franklin, S. (2003). Principios de administración. México: Editorial Continental.

Valenzuela, M. (2005). Indicadores de Salud: Características, Uso y Ejemplos. Revista Ciencia y Trabajo, 17, 118-122. 
